\title{
Influencia en los modelos del alisamiento de las impresiones de alginato con el dedo humedecido
}

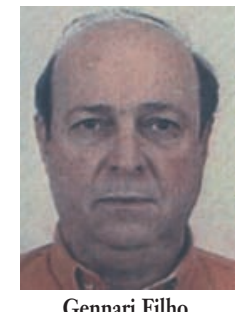

Gennari Filho, Humberto

\section{Influence of finger-smoothed moistening of irreversible hidrocolloid on dimension and surface}

\author{
changes of molds
}

\section{Gennari Filho, Humberito* Vedovalto, Eduardo*t Qưunelli Mazaro, José Vitor** Coelo Goidto, Marcelo* Dos Santos, Paulo Henrique*}

\footnotetext{
* Profesores del Departamento de Materiales Odontológicos y Prótesis de la Facultad de Odontología de Araçatuba-UNESP

** Doctorado del Curso de pos-graduación de Odontología-UNESP
}

\section{Correspondencia}

Humberto Gennari Filho

Facultad de Odontología de Araçatuba. Universidad Estadual Paulista "Júlio de Mesquita Filho». Departamento de Materiales y Prótesis. Rua José Bonifácio, 1193-Vila Mendoça Araçatuba-Sao Paulo-Brasil CEP:16015-050.

E-mail: gennari@foa.unesp.br
Resumen: El objetivo del estudio fue evaluar la presencia de burbujas superficiales y la alteración dimensional en los modelos obtenidos de impresiones de alginato, con y sin alisamiento con el dedo humedecido en agua. Se hizo una matriz en resina acrílica del maxilar desdentado cuyo paladar se modificó para que presentara una superficie llana y lisa y se insertaron 5 pernos metálicos en la cresta del reborde en lugares predeterminados. El estudio se dividió en 6 grupos de acuerdo con la técnica de impresión y del material usado (Jeltrate, Jeltrate plus e Hidrogum), con un total de 10 modelos para cada grupo. El paladar de los modelos se valoró con lupa (50 aumentos) lo que permitió la estimación de las burbujas. La lectura de los modelos se hizo por digitalización de los modelos, midiendo la distancia entre los pernos con el software AutoCAD 2000. Los datos según el análisis de la varianza (ANOVA) y el test de Tukey (5\%) indicaron una reducción significativa en el porcentaje de burbujas cuando se hacia el alisamiento. Los modelos donde se hizo el alisamiento presentaran alteraciones sin diferencias estadísticamente significativas respecto a los grupos donde no se hizo el alisamiento. El análisis de los resultados permitió concluir que el alisamiento del alginato con el dedo humedecido no afectó la precisión de los modelos del yeso en ningunos de los alginatos probados. El alisado dio lugar a una reducción significativa en el número de burbujas superficiales.

Palabras clave: Impresiones. Alginato. Alisamiento. Modelos.

Abstract: The purpose of this study was to evaluate the presence of superficial bubbles and dimensional changes of three types of irreversible hydrocolloids smoothened with a moist finger and not smoothened. A master die was made with acrylic resin, representing an edentulous maxillary palate that was modified in order to provide a flat surface. Five pins were inserted in the alveolar crest at predetermined areas. The study was divided in 6 groups in agreement with the molding technique and the hydrocolloid material (Jeltrate, Jeltrate Plus and Hidrogum). These consisted of 10 impressions for each group. The palate region of the molds was assessed with a microscope (50x) to allow bubble scoring. The mold was immediately poured with type IV dental stone. The casts were scanned and digitalized. The distances between pins were measured employing AutoCAD 2000. The data was submitted to ANOVA and Tukey's test with significance level of 5\%. There were no significant differences between cast accuracy obtained from either impression technique regardless of the alginate tested. From the results obtained it was concluded that the presence of bubbles was significantly higher $(p<0.05)$ in the groups that water rinsing with was not performed; the alginate "Jeltrate» showed a larger discrepancy between both techniques (with and without moist finger smoothening).

Key words: Dental impression materials. Alginates. Dental impression technique. Dental models.

\section{BIBLID [1138-123X (2007)12:1-2; enero-junio 1-104]}

Gennari Filho H, Vedovatto E, Quinelli Mazaro JV, Coelho Goiato M, Dos Santos PH. Influencia en los modelos del aislamiento de las impresiones de alginato con el dedo humedecido. RCOE 2007;12(1-2):55-61. 


\section{Introducción}

Frecuentemente se observa en la clínica diaria la no adecuación de protocolos preestablecidos respecto a la manipulación y al uso de los materiales odontológicos. Incluso con resultados inmediatos satisfactorios, el uso habitual de las técnicas no presupone su aceptación, requiriendo investigaciones científicas para validarlas. Morris et al ${ }^{1}$ relataron la posibilidad de mejorar la superficie de los modelos de yeso a través de impresiones de alginato alisadas con agua antes de la impresión, no obstante no evaluaron si el acto podría afectar a la precisión dimensional.

Lim et $\mathrm{a}^{2}$, encontraron una mejor superficie del modelo analizando solamente un tipo de alginato, sin embargo es interesante comprobar si otras formulas de hidrocolóides irreversibles se comportan de la misma manera después de alisarlas con agua antes de la toma de impresión.
La pérdida de precisión y la distorsión de los modelos que ocurre en la clínica, son directamente proporcionales a la apetencia que los alginatos tienen por el agua ${ }^{3,4}$, de manera que la incorporación de mas o menos agua puede repercutir considerablemente en la fidelidad de los modelos 5 . Por lo tanto, debido a que está cuestionado el alisamiento con agua conseguir modelos con una calidad superficial mejor, asi como que la incorporación de agua puede modificar la precisión dimensional de los modelos, el objetivo de este estudio fue valorar la calidad superficial de los modelos conseguidos con impresiones con y sin alisado con agua, con tres tipos de alginato, asi como las alteraciones dimensionales de los modelos de yeso.

\section{Material y Método}

Se construyó un modelo en resina termopolimerizable (QC-20, Dentsply), de un maxilar sin dientes cuyo paladar se modificó para conseguir una superficie llana y lisa, y con 5 pernos metálicos (sección de 1mm) insertados con un paralelizador en la cresta del borde, en lugares predeterminados (Figura 1). Estos cinco pernos metálicos delimitan dos figuras geométricas triangulares que se utilizaran como referencia para las medidas (Figura 2).

La muestra son 6 grupos con 10 especimenes cada uno, de acuerdo con el material de impresión usado (Jeltrate, Jeltrate Plus e Hidrogum) y de dos técnicas de impresión:

- Técnica 1 - el alginato se mezcla manualmente y se carga la cubeta solamente con la espátula.

- Técnica 2 - similar a la técnica 1 , pero alisando el alginato con el dedo humedecido en agua hasta conseguir una superficie lisa.

Las impresiones se hicieron con una cubeta en acrilico autopolimerizable perforada, para que el material de

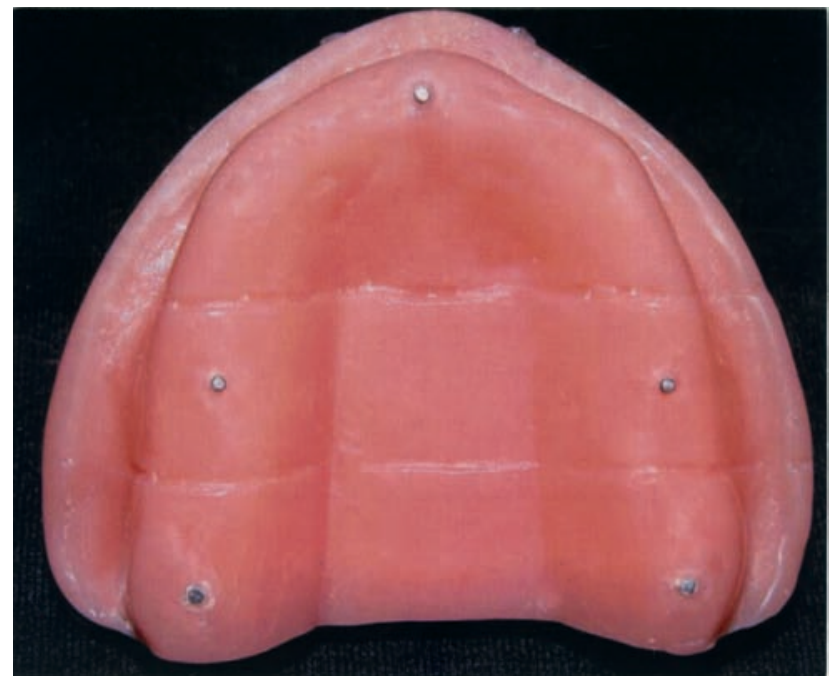

Figura 1. Matriz en resina acrílica con los pernos de referencia.

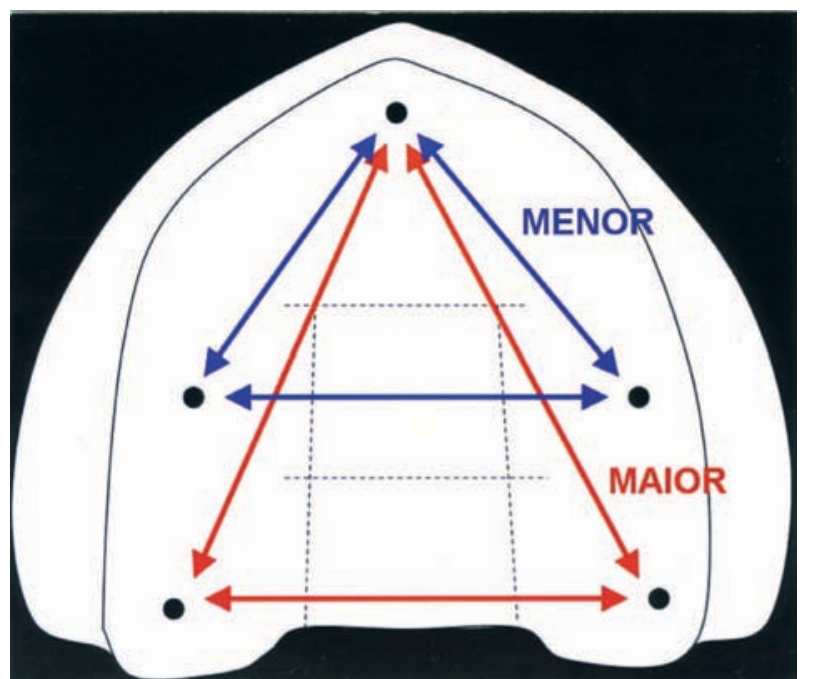

Figura 2. Situación de los pernos y formación de dos triángulos. La zona trazada indica la lectura de las burbujas. 
impresión tuviera un espesor uniforme (aproximadamente $3 \mathrm{~mm}$ ), según lo recomendado por Phillips y Ito $^{6}$. Figura 3.

La cubeta se colocó hasta que sus bordes coincidieron con los laterales del modelo, limitando así la profundidad de la impresión y permitiendo el vaciado uniforme del material.

Para el análisis de la superficie del alginato se observó un área de 3,0 x $1,5 \mathrm{~cm}$ en la región correspondiente al paladar del modelo (Figura 4), a través de una lupa (Carl Zeiss - Citoval 2, Alemania), de 50x, para medir la cantidad de burbujas presentes (Figuras 5 Y 6). A resaltar que el tamaño de las burbujas no era tenido en cuenta, solo el número, según lo preconizado por la metodología de Soh y Chong?.
Después de la inspección, las impresiones fueron vaciadas en yeso tipo IV (Durone/Dentsply) mezclado al vacio (Turbomix EDG-equipamiento Ltda). Los modelos (Figura 7) se escanearon y fueron digitalizados con el scanner de mesa (HP-6100C), con la captura de la imagen con definición de 600 ppi. Todas las imágenes se midieron con el software AutoCAD

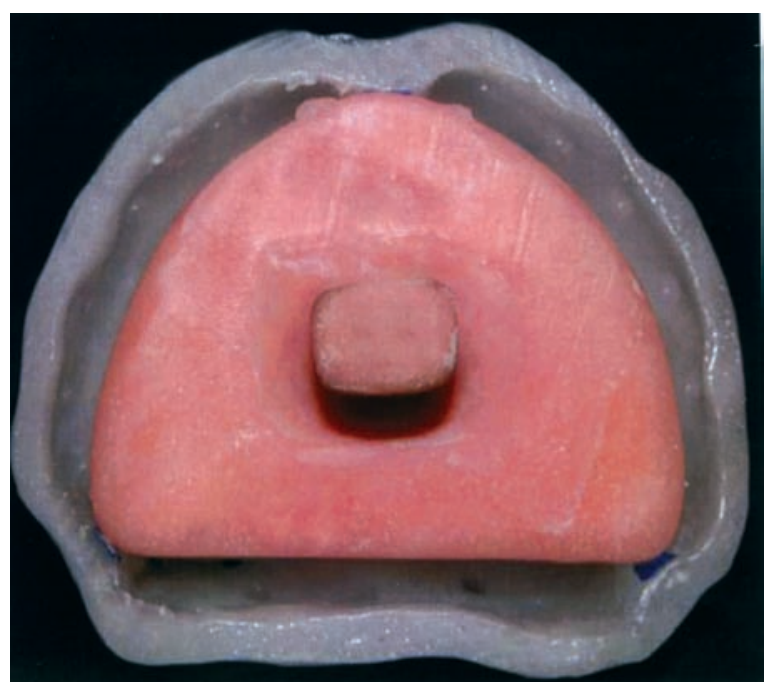

Figura 3. Cubeta/matriz.

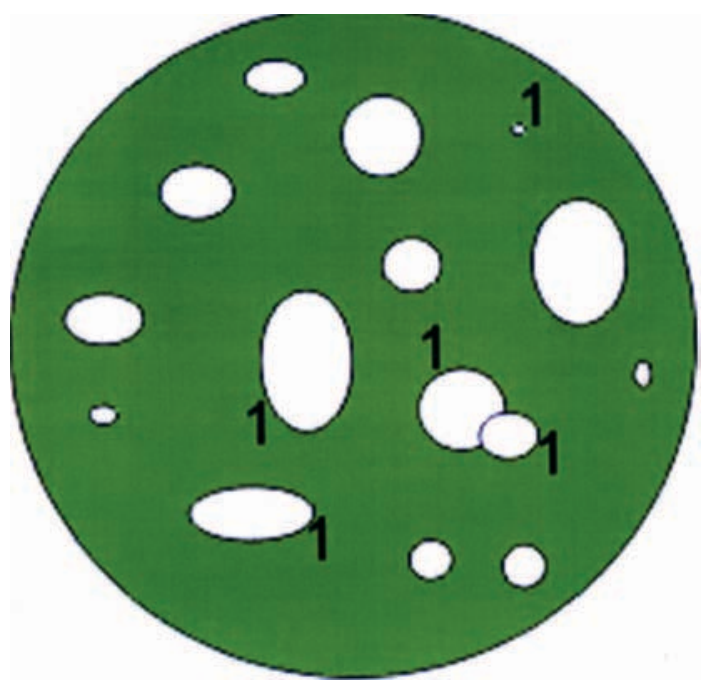

Figura 5. Representación esquemática del área visual $y$ del criterio de lectura.

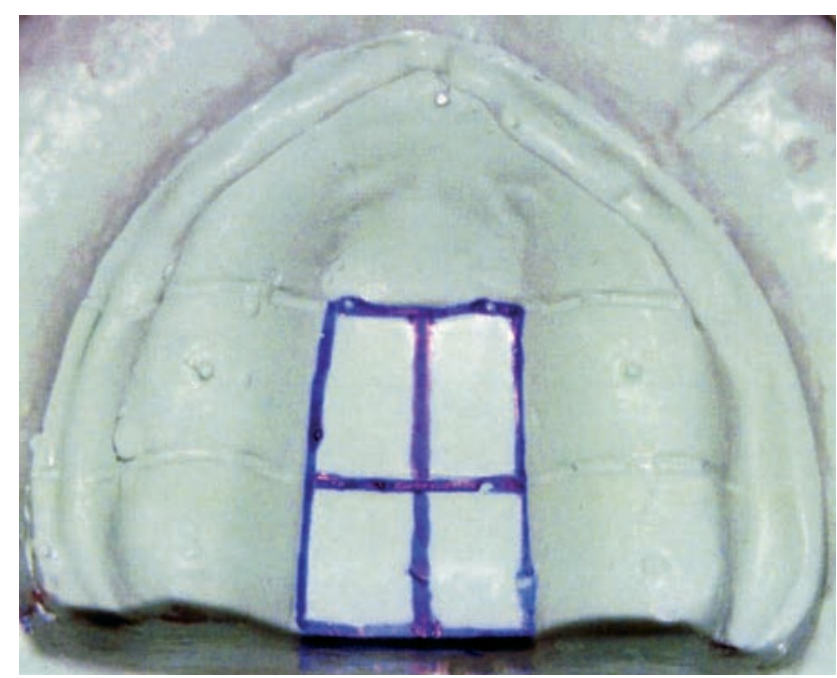

Figura 4. Impresión de alginato y delimitación del área de lectura.

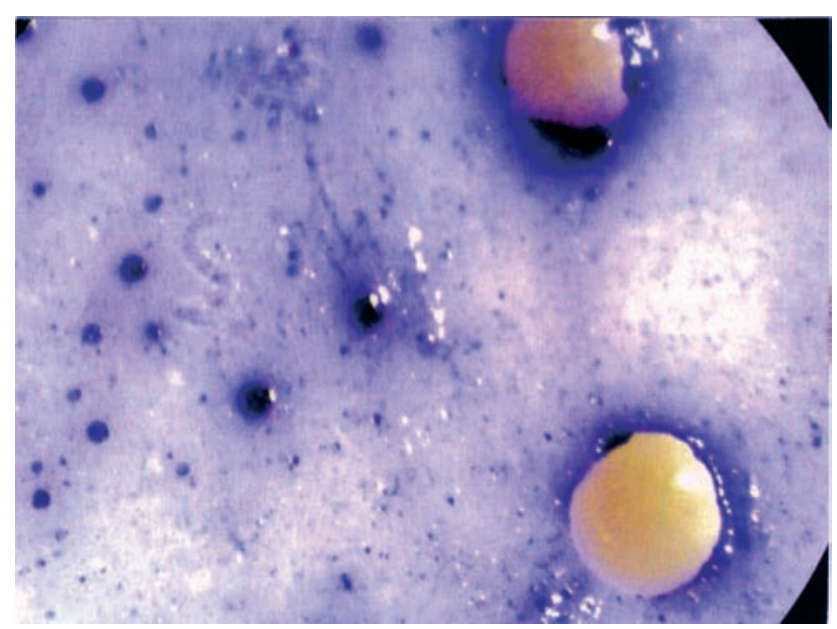

Figura 6. Imagen de las burbujas a 50 aumentos.. 


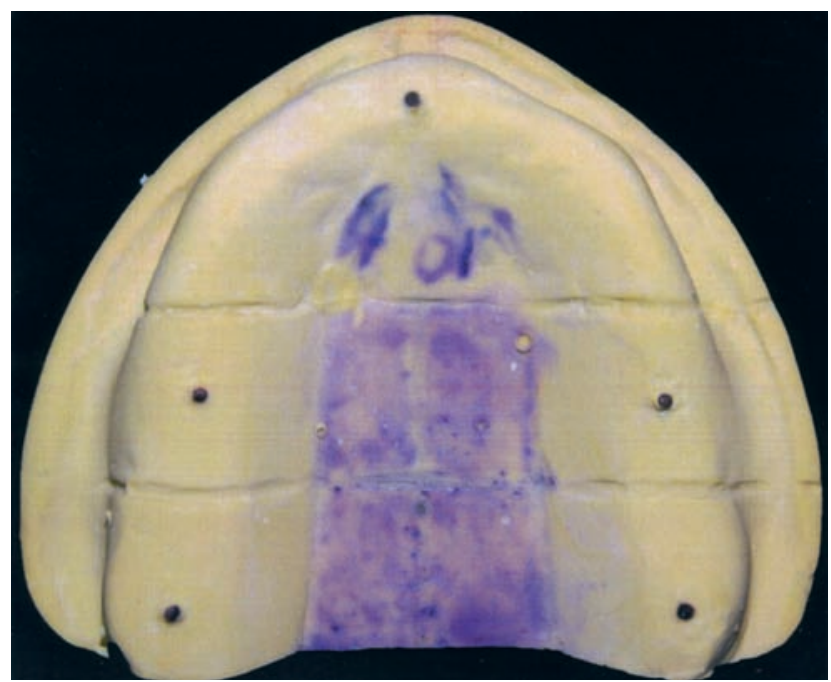

Figura 7. Imagen del modelo de yeso.

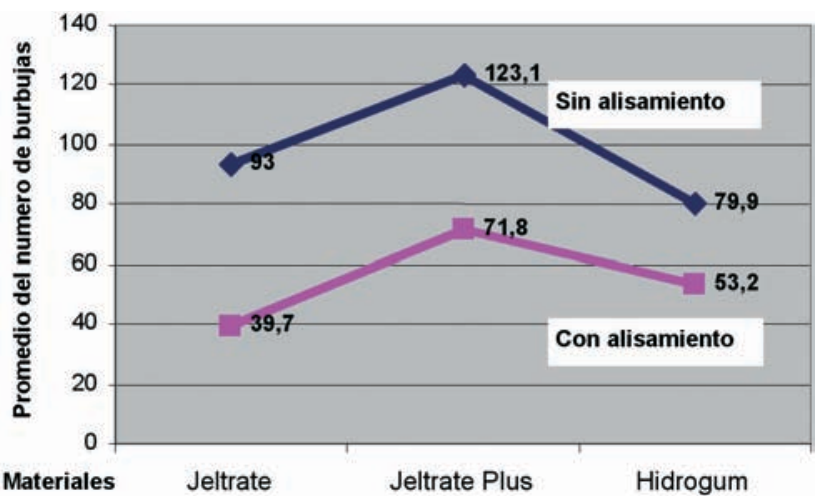

Figura 9. Comparación del número de burbujas en relación a los alginatos.

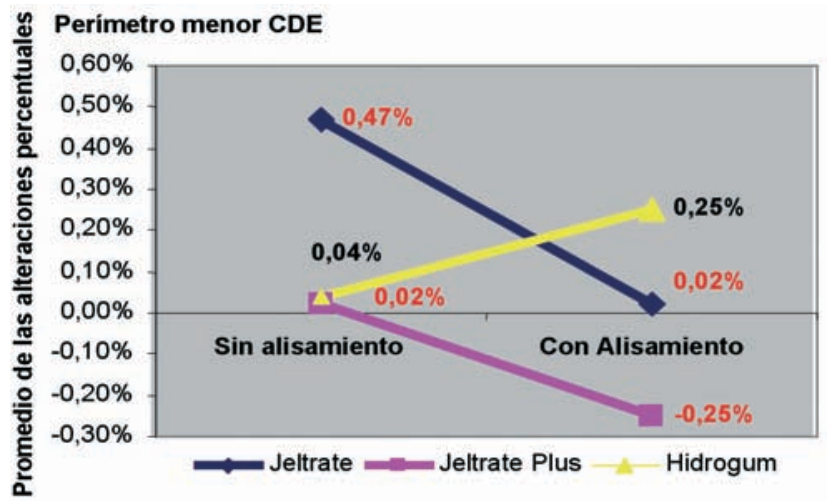

Figura 11. Comparación de la alteración dimensional en los grupos examinados para el perímetro del triángulo menor

$(C D E)$ en relación a la matriz.

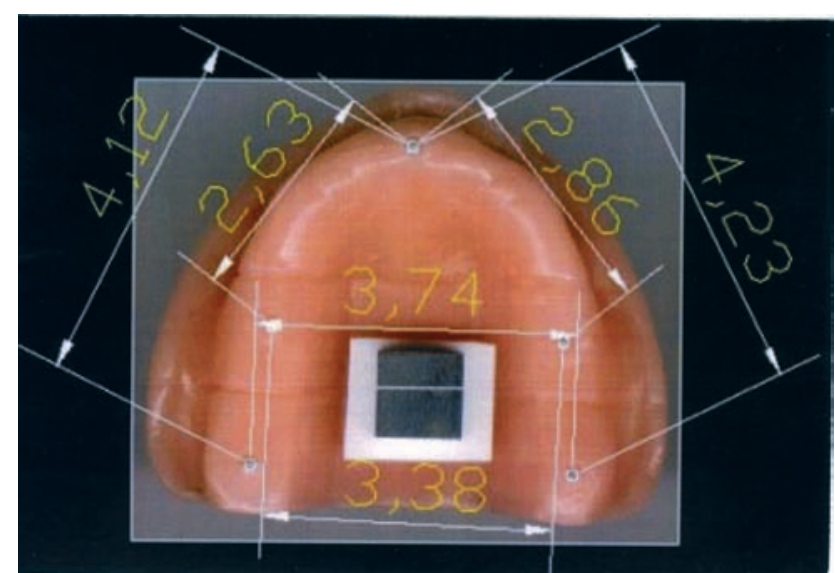

Figura 8. Imagen de la matriz para medir con programa AutoCAD 2000.

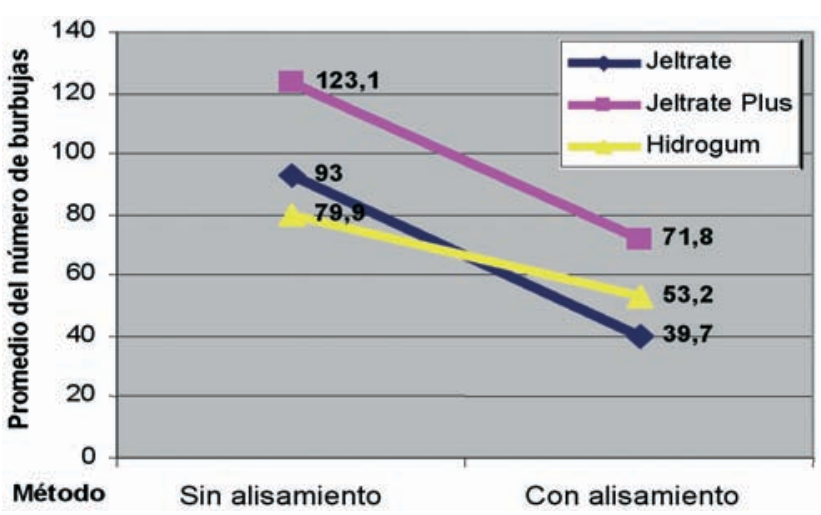

Figura 10. Comparación del número de burbujas con relación a la técnica de alisamiento.

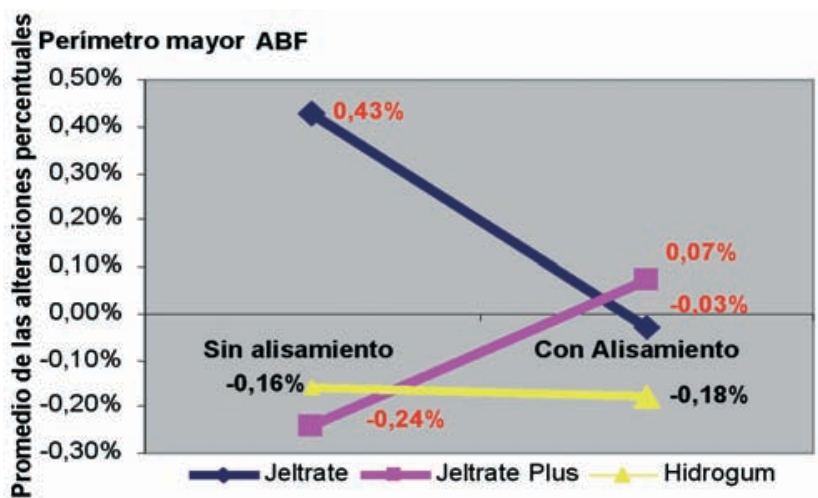

Figura 12. Comparación de la alteración dimensional en los grupos examinados para el perímetro del triángulo mayor $(A B F)$ en relación a la matriz. 
2000 para la técnica del análisis de las imágenes (Cennari Filho et al. 2003), asi fué posible medir el perímetro de los triángulos mayores y menores (Figura 8). Cada imagen se midió tres veces en días distintos. A señalar que todos los modelos fueran digitalizados en un bloque metálico de $10 \times 10$ milímetros, que sirvió como referencia para la estandardización de todas las imágenes en la lectura (Figura 8), según lo que fue propuesto por Cennari Filho et $a l^{8}$.

Se hizo un a estadistica descriptiva e inferencial con un analisis de la varianza y el test de Tukey.

\section{Resultados}

\section{Análisis de la superficie de los modelos}

La Tabla I muestra el tratamiento estadístico del análisis de la varianza para la cantidad de burbujas en los grupos, indicando la diferencia estadísticamente significativa para la comparación entre los tres alginatos y el alisamiento.

Las figuras 9 y 10 muestran el promedio de burbujas en relación a los alginatos y al alisamiento.

\section{Análisis de la alteración dimensional de los mode- IOS}

Los resultados de las medidas de los modelos de yeso para todas las técnicas se compararon con las medidas de la matriz en alteraciones porcentuales. Los valores de los triángulos que habían sido analizados (mayores y menores) fueran sometidos al tratamiento de análisis de la varianza y al test de Tukey $(5 \%$ de significa-

\begin{tabular}{|c|c|c|c|c|}
\hline Causas de la variación & G.L. & S.Q. & Q.M. & $\mathbf{F}$ \\
\hline Material/alginatos & 2 & 32.2327 & 16.1164 & $5.4320 * *$ \\
\hline Técnica del alisamiento & 1 & 100.6598 & 100.6598 & 33.9274 ** \\
\hline Mat.x alisamiento & 2 & 6.9306 & 3.4653 & $1.1680 \mathrm{NS}$ \\
\hline Tratamientos & 5 & 139.9231 & 27.9464 & \\
\hline Residuo & 54 & 160.2134 & 2.9669 & \\
\hline
\end{tabular}

\begin{tabular}{|c|c|c|c|c|}
\hline Causas de la variación & G.L. & S.Q. & Q.M. & $\mathbf{F}$ \\
\hline Material/alginatos & 2 & 1,3699 & 0,685 & $6,3345^{* *}$ \\
\hline Técnica de alisamiento & 1 & 0,4318 & 0,4318 & $3,9935 \mathrm{NS}$ \\
\hline Mat. $x$ alisamiento & 2 & 1,1608 & 0,5804 & $5,3676^{* *}$ \\
\hline Tratamientos & 5 & 2,9625 & 0,5925 & \\
\hline Resíduo & 54 & 5,8388 & 0,1081 & \\
\hline
\end{tabular}

\begin{tabular}{|c|c|c|c|c|c|c|c|c|}
\hline \multirow[b]{2}{*}{ Jeltrate } & \multicolumn{3}{|c|}{ Sin alisamiento } & \multicolumn{3}{|c|}{ Con alisamiento } & \multicolumn{2}{|c|}{ General** } \\
\hline & $0,47 \%$ & A & a & $0,02 \%$ & $A B$ & b & $0,25 \%$ & A \\
\hline Jeltrate Plus & $0,02 \%$ & B & a & $-0,25 \%$ & B & a & $-0,11 \%$ & B \\
\hline Hidrogum & $0,04 \%$ & B & a & $0,25 \%$ & $A$ & a & $0,14 \%$ & A \\
\hline General *** & $0,18 \%$ & & a & $0,01 \%$ & & a & & \\
\hline \multicolumn{9}{|c|}{$\begin{array}{l}\text { Los promedios seguidos por las letras mayúsculas en la columna y las letras pequeñas en } \\
\text { la filas se diferencian entre sí en el nivel de significación de } 5 \% \text {. } \\
\text { **. Independiente de la técnica } \\
\text { ***. Independiente del tipo de material }\end{array}$} \\
\hline
\end{tabular}

ción). Las tablas II y III que expresan el análisis del conjunto de las alteraciones para el triángulo menor muestran que hay diferencias estadísticamente significativas entre los diversos tipos de alginatos utilizados y no para el alisamiento.

Las tablas IV y $\vee$ que expresan la evaluación de las alteraciones en el triangulo mayor, muestran el mismo 


\begin{tabular}{|c|c|c|c|c|}
\hline \multicolumn{5}{|c|}{$\begin{array}{r}\text { Tabla 4. Análisis de la varianza para la allera } \\
\text { en el perímetro mauor flf }\end{array}$} \\
\hline Causas de la variación & G.L. & S.Q. & Q.M. & $\mathbf{F}$ \\
\hline Material/alginatos & 2 & 1,4859 & 0,743 & $8,4781^{* *}$ \\
\hline Técnica de alisamiento & 1 & 0,0482 & 0,0482 & $0,5496 \mathrm{NS}$ \\
\hline Mat. X alisamiento & 2 & 1,4914 & 0,7457 & $8,5096 * *$ \\
\hline Tratamientos & 5 & 3,0255 & 0,6051 & \\
\hline Resíduo & 54 & 4,7921 & 0,0876 & \\
\hline
\end{tabular}

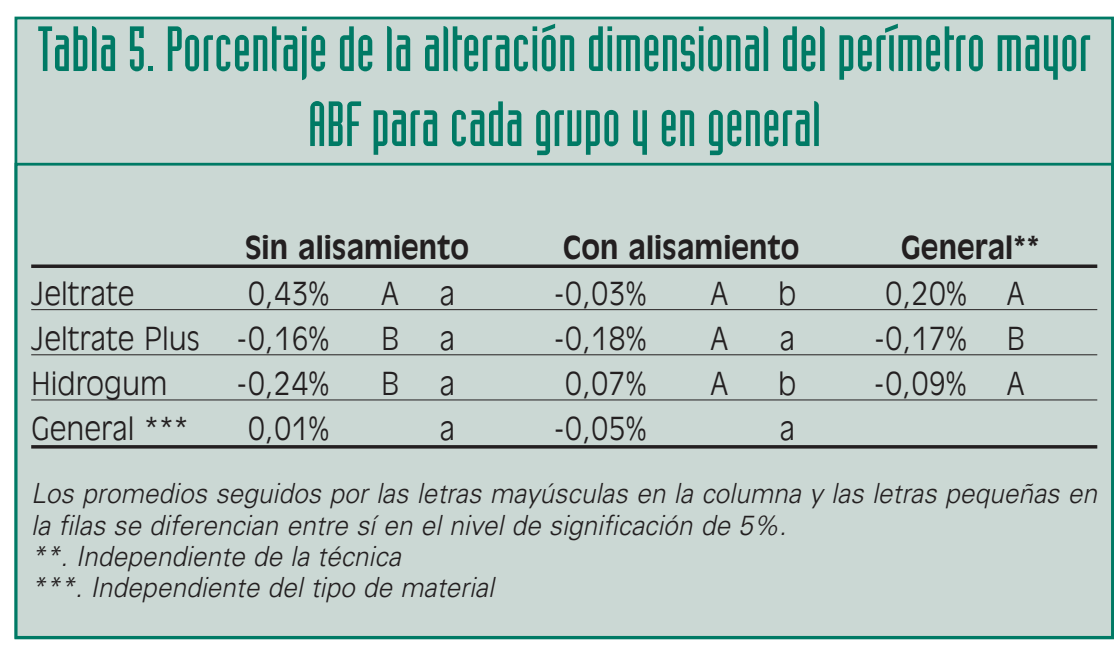

comportamiento de las alteraciones anteriores (triángulo menor); hay diferencias estadísticamente significativas entre los alginatos y no entre las técnicas de alisamiento.

Las figuras 11 y 12 representan respectivamente las alteraciones de la técnica sin y con alisamiento en cada alginato probado para el triángulo menor y mayor respectivamente.

\section{Discusión}

Como se relata en estudios anteriores $^{1,2}$, la técnica del alisamiento influyó directamente en la calidad superficial del molde. Nuestros datos muestran que cuando se carga la cubeta y se alisa con el dedo humedecido en agua, se reducen las burbujas y irregularidades en más del $50 \%$. Aunque la magnitud de la reducción varia entre los alginatos estudiados, todos sin excepción, exhibieron mejoria en la calidad del modelo lo que implica obtener modelos de yeso de mejor calidad. Sin embargo, la calidad superficial no es la única cuestión importante para la construcción de las prótesis, sino que tambien hay que valorar la fidelidad dimensional que
Ios materiales/alginatos, sobre todo cuando se tratan las impresiones de una u otra manera ${ }^{9}$.

Nuestros datos indican que independiente del lugar del análisis (triángulo menor o mayor), no hay diferencias estadísticamente significativas entre las técnicas de impresión, cuando no se considera el tipo de material. sin embargo hay diferencias estadísticamente significativas para las menores distorsiones para la técnica de alisamiento. Esto implica que alisar con el dedo humedecido no afecta la precisión dimensional de los modelos y que en algunos casos optimizan la precisión de los mismos en comparación con la matriz. Hay que considerar que las alteraciones de los modelos de alginato no se ven influenciados solamente por factores inherentes al material sino también por la influencia de las retenciones del área medida que pueden inducir grandes tensiones y también por la capacidad de recuperación elástica del material que pueden provocar alteraciones permanentes ${ }^{10}$.

Por otro lado aunque la adherencia superficial puede ser influida por el agua depositada durante el alisamiento, se considera esto un factor favorable que puede explicar los mejores resultados conseguidos por esta técnica, como demuestran Vassilakos $Y$ Fernandes en relación a los elastómeros.

Cuando se considera el tipo de material para la técnica en que no se efectuó el alisamiento, se observan alteraciones estadísticamente significativas tanto para el triángulo menor como para el mayor, entre el Jeltrate en relación al Jeltrate Plus y Hidrogum que presentaron alteraciones 
menores. Cuando se hace alisamiento solo hay diferencias estadísticamente significativas para el triángulo menor entre el Hidrogum (0,25\%) y Jeltrate Plus $(-0,25 \%)$ que exhibieron alteraciones semejantes pero de dirección inversa. Hay que decir que muchas de estas alteraciones ocurren en función de la susceptibilidad del alginato a las condiciones externas y estamos de acuerdo con Frank et $\mathrm{al}^{12}$ en que la variabilidad en las impresiones con alginato puede relacionarse con la destrucción y la pérdida de material durante la extracción del modelo.

\section{Conclusiones}

En función de los análisis de nuestros resultados y considerando las limitaciones de los estudios «in vitro» podemos concluir:

1. La técnica del alisamiento con el dedo humedecido en agua consigue modelos con un número perceptiblemente menor de burbujas.

2. No hay diferencias estadísticamente significativas entre las alteraciones de los modelos obtenidos por la técnica sin alisamiento en relación a la técnica donde sí se utilizó.

\section{Bibliografia recomendada}

Para profundizar en la lectura de este tema, el/los autor/es considera/an interesantes los artículos que aparecen señalados del siguiente modo: *de interés ** de especial interés.

1. Morris JC, Khan Z, Fields HJR. Effect on surface detail of casts when irreversible hydrocolloid was wetted before impressions making. J Prosthet Dent 1983;3:328-30.

2. Lim PF, Neo KH, Sitoh L, Yeo KL, Stokes A. Adaptation of finger-smoothed irreversible hydrocolloid to impression surfaces. Int $\mathrm{J}$ Prosthodont 1995;2:117-121.

3. Christensen GJ. Impression materials for complete and partial denture prosthodontics. Dent Clin North Am 1984;2:223-237.

4. Anusavice, KJJ. Phillips. Materiais Dentários. $10^{\mathrm{a}}$ ed. Rio de Janeiro: Guanabara Koogan, 1998.

5. Cohen BI, Pagnillo M, Deutsch AS, Musikant BL. Dimensional accuracy of three different alginate impression materials. J Prosthodont 1995;3:195-199.

6. Phillips RW, Ito BY. Factors influencing the accuracy of reversible hydrocolloid impressions. J Am Dent Assoc 1951;1:1-17.

7. Soh G, Chong YH. Determination of na optimal magnification for examining voids in elastomeric impressions. Int $\mathrm{J}$ Prosthodont 1990; 6:73-576.

8. Gennari Filho H., Vedovatto E., Lazari J.A.B., Assunção, W.G., Shibayama, R. Avaliação comparativa da posição relativa dos dentes artificiais entre três métodos de inclusão de próteses totais polimerizadas em banho de água quente. Cienc Odontol Bras. 2003;4:32-40.

9. Nakagawa H, Hiraguchi H, Uchuda H, Tanabe
N. Effect of rinsing hydrocolloid impressions using acid electrolyzed water on surface roughness and surface hardness of stone models. J Oral Sci 2002;3-4:141-146.

10. Millar BJ, Dunne SM, Robinson PB. The effect of a surface wetting agent on void formation in impressions. J Prosthet Dent 1997;1:54-56.

11. Vassilakos N, Fernandes CP. Effect of salivary films on the surface properties of elastomeric impression materials. Eur J Prosthodont Restor Dent 1993;1:29-33.

12. Frank RP, Thielke SM, Johnson GH. The influence of tray type and other variables on the palatal depth of casts made from irreversible hydrocolloid impressions. J Prosthet Dent 2002;1:15-22.0 